\title{
RANDOM PROCESSES AND THEIR APPLICATION TO DEMOGRAPHY AND INSURANCE ${ }^{1}$ )
}

\author{
B. W. GNedenko \\ U.S.S.R.
}

\section{Mr. Chairman, Honourable Colleagues!}

Let me thank the organisers of this symposium for the honour they have done me by inviting me to present my report before this eminent audience. I would also like to express my thanks to all who are present here for the confidence shown to me as rapporteur.

The genesis and development of the theory of random processes is closely related to the problems of demography and insurance. Numerous problems stimulating the development of the theory of probability and the theory of random processes - the latter constituting one of the essential parts of the modern theory of probability-are connected with these two fields of research and activity.

The theory of random processes, being stimulated-similarly to other fields of mathematics-by concrete practical tasks, has created the general ideas and methods of research, achieving many valuable results. With the passage of time, it appeared that the theory of random processes can be usefully applied, by its conceptions, results and methods, to a number of scientific fields and practical activity. Its conceptions have found wide and important application to the earlier crystallized fields of human knowledge, as well as to the newly appearing ones.

These new fields of mathematics serve us as an example which once more corroborate the thesis, well known to us, according to which the course of the development of human knowledge starts from empirical observations, then passes up to abstract thinking and finally to practice.

Practice, in turn, after receiving new scientific stimulus and possibilities of improvement, puts before science new and fascinating tasks which require its further development. In recent years, the

1) Invited lecture to open the 8th Astin Colloquium held at Sopot, Poland, 22-24 september, 1969 . 
theory of random processes was developing under practical influence in a number of fields, from among which I shall select here only a few ones, which confirm, in my opinion, the particular advantages of this theory for both the theory and practice in the field of insurance and demography.

As is known, under the term "random process" we understand the function of one variable (scalar and vectoral). This variable is usually defined by the letter " $t$ ", being called "time". The number of inhabitants of a given town constitutes the simplest example of a random process. In fact, this number is subjected to random changes in time because of the increasing and decreasing number of the inhabitants resulting from births and deaths occurring in the town under consideration. The course of the process we are interested in is fully expressed by one figure. If, moreover, we are also interested in the division of the inhabitants according to sex, we should examine a two-dimension random process $[\mu(t), \gamma(t)]$ in which $\mu(t)$ and $\gamma(t)$ define the respective numbers of women and men at the moment " $t$ ". The investigation concerning the changes in the number of inhabitants of the town, taking into consideration their age-distribution forces us to examine the process with a greater number of variables.

In the case of more complicated processes, the state of the system cannot be described by means of only few variables. In addition, there exist many situations in which the state of the system requires to be described not only by means of figures but also from the point of view of its features. Such a means of description is needed in medicine (but, of course, not only in medicine), for the account of the patient's illness. By way of example, in the case of a cardiac disease, the degree of the patient's skin lividity or the presence of a swelling of his eyelids are fairly important in this respect. For this reason, according to the modern theory of probability, we examine the random processes defined by the elements of an arbitrary abstract set. Such a broader approach is advantageous with regard to all branches of applied science, including insurance and demography. This aspect will be analysed later.

Application of the conception of random process, in its broadest sense and with no additional restrictions concerning its course, would not be possible or could lead to no significant theoretical 
result or be useless from the point of view of its practical application. This is why, since the very beginning of the development of the theory of random process elements, the necessity has been to isolate - on the basis of problems which aimed at giving the random processes a concrete significance-the separate classes of these processes. The processes which received the name of "birth and death processes" constituted the first, chronological class of processes, which was subjected to precise and sufficiently universal tests, at the beginning carried out on particular examples concerning biological, physical and technical problems. Let us assume that the system interesting us can find itself, at any moment, in only one from the states: $E, E_{1}, E_{2}, \ldots$ the set of which is limited and countable. The probability of passage from the state $E_{n}$ to the state $E_{n+1}$, in a short period of time $h$, is equal to $\lambda_{n}(h)+o(h)$ and to the state $E_{n-1}, \gamma_{n} h+o(h)$. It is assumed that the probability of passage from the state $E_{n}$ into an optional state $E_{n+k}$ when $k>\mathrm{I}$, is infinitely small as compared with $h$.

Defining by $P_{n}(t)$ the probability of the fact that at the moment $t$ structure of the system finds itself in the state $E_{n}$, we can see that functions $P_{n}(t)$ can be defined by the following equations:

$$
P_{0}^{\prime}(t)=-\lambda_{0} P_{0}(t)-\gamma_{1} P_{1}(t)
$$

and when $k \geqslant \mathrm{I}$

$$
P_{k}^{\prime}(t)=-\left(\lambda_{k}+\gamma_{k}\right) P_{k}(t)+\lambda_{k-1} P_{k-1}(t)+\gamma_{k+1} P_{k+1}(t)
$$

It is obvious that for a full explanation of the meaning of $P_{n}(t)$ it is necessary to show the initial state, from which the system generates.

Naturally in the case of arbitrary $k$ and $t$ the function $P_{k}(t)$ must not be negative: $P_{k}(t) \geqslant 0$.

It can happen that for a certain $t$

$$
\sum_{k=0}^{\infty} P_{k}(t) \neq \mathrm{I}
$$

It means that for this value of $t$ with probability finite and different from zero, the system passes to the state $E_{\infty}$.

This case receives a very simple interpretation in the processes of pure propagation $\left(\gamma_{k}=0\right.$, for all $\left.k \geqslant \mathrm{I}\right)$. Let us imagine that the state $E_{k}$ means the existence of elements $k$ in a certain set. 
The inequality ( $\mathrm{I}$ ) can be interpreted in the following way: the sum of the probabilities that in the set, at the moment $t$, are some elements $k$ is smaller than unity; it means, that with the probability complementing this figure up to unity, the set comprises the infinite number of elements. In the phenomena of radioactive disintegration such a possibility is treated as an explosion.

At the present time the processes of birth and death find wide application to various concrete investigations: in the theory of reliability, the theory of queues (theory of mass service), biology, economics, demography and the like. However, the hypothesis (assumptiones) made with regard to the examined process and its course are fairly limiting. It particularly refers to the pure processes of birth if we assume that at the moment $\mathrm{T}=0$ the system finds itself in the state $E_{0}$, the following equation applies: $P_{0}(t)=e^{-\lambda_{0} t}$

This equation particularly means that the prohability distribution of the length of the period during which the system finds itself in the state $E_{0}$ is an exponential distribution. Thus, the following fact results from it as a conclusion: if the system found itself in the state $E_{0}$ during a certain period of time $\tau$, the probability of the assumption that the system will remain in this state during the time $t$ does not depend on $\tau$.

Let us imagine now that the system subjected to our analysis constitutes the group $\mathrm{N}$ of persons of a definite age of the group of identical machines working in the same way. The state $E_{k}$ means that in this group the number of $k$ persons ( $k$ machines) have lost their productive capability. Assuming that the development of the system interesting us is subjected to the law by which the processes of births and deaths are directed, we must assume that independently of the period during which the work was going on in our system without losing the working capability, the probability of the state $E_{0}$ at least during the period of time t remains equal to $e^{-\lambda_{0} t}$. We know that in reality such an argumentation is in disagreement with the true situation: both people and machines wear off and grow old.

In the theory of probability, first of all, in connection with the problems of physics, processes of a wider type, namely the so called Markov processes, started to be examined. The essential contribution to the development of the theory of Markov processes was made 
by such eminent scientists as: A. N. Kolmogrow, P. Levy, W. Feller and others. No restrictions are made with regard to the set of possible states of the system under examination in the theory of Markov processes. Such set can be discrete, just as is the case in the theory of birth and death; it can be continuous or can constitute an arbitrary, abstract set. This set of possible states will be defined by us by $E$, and its separate elements-by $x, y, \ldots$, while $A, B, \ldots$ will define its possible subsets for which the probability is defined that the process $\xi(t)$ will find itself at this moment in the state belonging to the given subset.

Let us notice that application of mathematical methods to the analysis of real phenomena: social, biological, physical, economic or technical, requires the accomplishment of their initial schematization, or-as we say today - the construction of the models of their implementation. This results in the fact that:

at first, a mathematical analysis can be applied to the processes of changes in the certain systems only in the case when the assumption that any of their states can be fully represented with the help of one or other mathematical means was previously confirmed.

secondly: Any real process is usually so much complicated that attempts of describing it in its full generality and with all details can be considered, in advance, as a failure. An explorer is forced to simplify the phenomena, to reject certain elements, which in the given state of examination can be considered by him as unimportant. Thus a mathematician is forced to create the model of the phenomena examined by him and to ignore a number of relations and interesting details. Such a model, of course, only a scheme of the real process permitting, however, to describe its course with a relatively sufficient approximation.

This is a picture we are faced with, let us say, in classic mechanics, when we assume that the motion of a material body can be replaced by an analysis of a material point and that the properties of the motion of a material points system can be completely described by showing their position and velocity at any moment of time. In addition, it is assumed that the state of the system described in this way at any moment of time $s$, will fully define its state at the following moments $t \geqslant s$.

It has already been sufficiently well explained that the model 
of the occurring phenomena which is expounded by classic mechanics has, though a fairly great, all the same only a limited importance. In modern physics, technology, production, economics and social science we are faced, in principle, with a much more complex situation, when the state of the system at any moment of time no more defines synonymously the state of the system at the following moments of time. The further evolution of the system can be undertaken in different ways. In a number of very important and interesting cases, however, it appears that the course of phenomena can be excellently defined by the following reasoning: the knowledge of the state of the system at the moment $s$ defines not the state in which the system will find itself at the moment $t>\mathrm{s}$, but the probability of the fact that the system will be in one of the states of one subset or another. If we, moreover, assume that additional states of the system at the moment $\tau<s$ do not change this probability, we then say the analysed process is the one with no consequences or, which is more often met, that it is a Markovtype process.

The hypothesis that this is a Markov-type process is a fairly strong one but the change of the definition of the state permits to transform any random process into Markov-type one.

It should be said that transformation of a certain process into the Markov-type one is not always effective especially if it complicates the model to such an extent that the obtained advantages will become of no importance. The advantage of Markov-type processes consists, however, in the fact that good mathematical methods of investigation have been developed for them. Broadly speaking, the solution of equations connected with Markov processes can be brought down to the solution of differential and differential-integral equations. At the moment the scope of the application of Markov processes is very wide. The problems generated by Brownian motion and diffusion which constituted the beginning of the development of Markov processes, are actually only a small part of the wide field in which the theory of Markov processes is applied. Problems connected with theory of communication, insurance, biology, demography, enterprise management provide wide possibilities for application of the concepts and results of the Markov-process theory. 
Despite the wide application of the theory of Markov processes to modern practice, a number of problems in the field of physics, radio-technics and geophysics has led to the necessity of examining two other classes of random processes belonging to the group of processes with consequences. First of all this is the group of stationary processes and the group of the processes with stationary increments.

The term "stationary" is applied to processes which, in the course of time, become homogeneous in a definite sense. More precisely, independently of the values $t_{1} t_{2}, \ldots, t_{n}$ and $u$ and integer, positive $n$, the distributions of two groups of variables $\xi\left(t_{1}\right), \xi\left(t_{2}\right), \ldots \xi\left(t_{n}\right)$ and $\xi\left(t_{1}+u\right), \xi\left(t_{2}+u\right), \ldots, \xi\left(t_{n}+u\right)$ are the same.

We are faced fairly often with this type of processes both in nature and technological processes. Let us examine the production of yarn, for example. The considerably diversified features of the raw-material, the length of yarn, its resistance, the size of the transverse cross-cut and various speeds with which the raw material is fed into machines together with a number of other factors cause considerable differences in the features of the yarn produced. It also appears that a given property of the yarn in an optionally chosen part of the skein does not permit the complete description of the yarn properties in any other part of the skein. However, if the process of weaving can be considered as stabilized, the random characteristics of the yarn represent the stationary random process.

The hypothesis on the stationary character of nature and of technological processes is fully justified. But as far as social phenomena are concerned, the hypothesis of their stationary character is often rather a doubtful one. In a number of cases the situation can be saved by adoption of the hypothesis about the stationary character not of the very process $\xi(t)$, but of its increment $\xi(t+u)$ -- $\xi(t)$. The processes with stationary increments have found wide application in a number of practical problems: in the theory of queues, the theory of reliability, geophysics and other fields, as well.

Independently of the great possibilities of application in the above-mentioned classes of random processes, practice puts forward further demands for isolating the ever new classes of these 
processes, which are characterized by these or other properties. We shall deal here with three types only, which in recent years have been subjected to intensive investigations in connection with the possibility of numerous practical applications.

The theory of renewal processes was set up in connection with tasks referring to the dynamics of population, the theory of queues, the theory of stocks and the like. We shall illustrate the details of this theory by the example of task connected with the breakdown and renewal of elements. Let us imagine that an element (for instance an electric bulb) started to work at the moment 0 . The period of work of this element constitutes a random variable with the distribution $F(x)$.

Once this element stops working it is immediately replaced by another one with the same properties concerning its operation. The replacing element is, in turn, replaced by another one, and so on. We meet the replacements of this type not only in productive installations. The main problem we are faced with in this respect consists in the definition of the number of such replacements which we shall be obliged to make up to the moment $t$. Especially, if we wish to prepare a group of specialists in a given field, we should fix how many replacements will be needed in the period of interest to us. We have a similar situation in elaborating the plan of production or purchase of spare parts of a definite type, for a given enterprise. Here, evaluation of the indispensable quantity of spare parts for a given period of time represents a problem.

The genesis of the theory of branch processes, which have found a wide application in nuclear physics and technology, in biology and chemistry in the course of two, three decades, is connected with a certain demographic problem, namely family degeneration.

I take the liberty of quoting the words expressed by Francis Galton, and printed in the introduction to the paper by Galton and Watson which appeared in 1874 : The extinction of families, which played an eminent role in history, constitutes a fact often noticed in the past. Various guesses have been made in this respect. Well known are numerous examples of families, which, because of their members' dispersion, were becoming less and less numerous or even completely vanished. This tendency is universal to such a degree that in order to explain it some hypotheses was hurriedly 
made, assuming that the increasing standard of living and intensification of intellectual activity is accompanied by a decreased fertility. On the other hand, however, Alfons de Candol has drawn attention to the fact that because of the functioning of a simple law of random processes, a considerable number of families is always subjected to degeneration. Thus we are unable to decide whether in the group of families under consideration, degeneration takes place as a result of decreased fertility.

Problems of families vanishing have not become less important today. It is often, however, that instead of human families' degeneration that of a definite animal or plant species, of physical elements or industrial enterprises is of interest to us.

The simplest problem concerning the theory of branching processes is the following: There exist elements able to produce other elements of the same type. The initial set of elements will be called by us " 0 " generation, that of their "children"- the first generation. The "grandchildren" of the " 0 " generation will be the second generation and so on.

The problem arises concerning the regularities to which the quantities of these families $X_{1}, X_{2}, X_{3} \ldots$ are subjected. Undoubtedly, it is indispensable to have some hypothesis concerning the relations between the quantities of these families, if one wants to find a solution for problems arising in this connection. We usually limit ourselves to the assumption that we have here to do with Markow processes. We sometimes succeed in keeping up this assumption through the introduction of some types of elements concerning different rate of fertility. The process of the behaviour of branch processes was very seldom examined in the past, because the rate of fertility is changing according to the age of its elements. This is just the situation we have to do with both in a human society and in industry where the prolongation of the period of installations' usefulness is dependent on their productive capacities.

No need to speak about the fact that the development of the theory of random processes and examination of their ever new classes appears to be the important result of scientific progress and of application of mathematical methods to various practically important situations.

In the course of time, practice is forced to apply the ever new 
mathematical methods of analysis and then, to put requirements before mathematics concerning the necessity of its new methods development. The essential goal of mathematics consists not only in the development of mathematical theories but, moreover, in listening to the voice of practice and in the creation of such criteria, methods and theories which are, indeed, indispensable for practical application. In addition, the theory should also take into consideration these specific properties of the analysed subject, which constitute its exclusive features. That is why we perceive, in the case of real tasks, the presence of phenomena which can be described, in a satisfactory way, be means of random processes. The problem concerning the processes which are able to give a satisfactory description of the phenomenon interesting to us from the mathematical point of view, must emerge here inevitably. I am convinced that a mathematician can not remain indifferent to the natural phenomena, to the essential properties of concrete problems he tries to resolve and which concern natural science, production and economic or social problems, as well, with no profound explanation of the heart of the matter, applying of mathematical apparatus of investigation can direct the explorer's efforts in a wrong direction and lead him to erroneous conclusions. In order to avoid such a situation, or, at least, to diminish considerably the probability of this sort of errors, it is necessary that the mathematician comes down from Olympus and, together with specialists in a given field, deals with the preparation of indispensable conditions for successful results of the research work, and that he penetrates the essence of the analysed phenomena selecting, on this basis, the suitable mathematical method, and, even, creates such a method, if needed.

In connection with the above I would like to cite here the words, pronounced by the founder of the Moscow University more than two hundred years ago, according to which to analyse nature's phenomena it is not a mathematician proficient in difficult calculus who is needed, but one who knows how to support the discoveries by means of mathematics and how to deduce an unknown truth by a precise and untendentious method.

I would like to pass now to one scientific discipline which is applicable to practice and which has developed with unusual speed 
in the period of the last twenty years. Its achievements are connected to a considerable extent with the application of the theory of random processes and which, in turn, have exercised a considerable influence on the development of some directions of research work concerning this theory. As the science about which I shall speak is closely connected with the problems of demography and insurance and has taken a number of its essential ideas from them, it has become able to exercise, in turn, certain influence on their further development. I have in mind the theory of reliability.

The theory of reliability has developed in the last fifteen to twenty-years' period being influenced by actual problems of modern technology. It has a number of common points both with the theory of random processes and demographic problems. As has been said above, the theory of reliability has taken a number of basic criteria from the mathematical theory of demography and the theory of random processes constitutes its logical foundation, on the basis of which its formulations and quantitative evaluations are made. At the moment the development of the theory of reliability is in the state in which a number of problems and formulations analysed by it can be successfully used, both in the practice of insurances and in demography.

Generally speaking mathematics is, of course, similarly to theory of random processes only a means of analysis for the theory of reliability. Its basic aim is to elaborate the means for increasing the efficiency of technical installations, prolongation of the period of the faultless operation of these installations, elaboration of the principles of useful application of ready articles as well as of the principles of the qualitative control of technological processes of production. However, the solution of these tasks requires inevitably profound, physicochemical investigations and, also the resolving of a number of new and interesting mathematical problems. For this reason the creation of precise criteria of the theory of reliability requires a standardization of typical, technical situations to be made and mathematics to be used, but, first of all, it requires application of the theory of probability in particular taking into consideration its essential section, namely the theory of random processes.

But even such original and basic conceptions of the theory of reliability as "break-down", could not be defined without using 
formulations of the theory of random processes. What should be understood, indeed, under the term "break-down" of a bulb? The burning through of the filament or the loss, even partial, of its lighting properties? It is known that in many practical situations the loss of lighting properties of the bulb, for instance in a lighthouse-lamp, in operating theatres etc., is inadmissible even to the degree of a few per cent. In every-day life, however, the electric bulbs are in principle used until the movement of filament blows. As can be seen from the above-mentioned examples, the term "break-down" refers to completely different events, though the problem concerns practically the same, from the technical point of view, goods.

Let us assume that, at any moment, the arbitrary technical system finds itself in the defined state .

The set of all its possible states will be called "its space" $\mathrm{X}$. In the case of technically different systems, we must, of course, examine different phase spaces, which generally are very complex in character. It is only in very seldom cases that we succeed in a satisfactory description of the phenomenon of the loss reliability, by means of a simple phase space. With the passage of time the state of the system is undergoing changes being, thus, a certain function of time. The change in the system in the function of time can be, then, presented by a "trajectory" in the phase space $X$. This trajectory describes the changes of the system in time.

On the basis of these or other criteria, the subset $A$ is isolated from the phase space $X$. However, penetration of the system into this subset is considered as inadmissible. In any case, if the state of the examined technical system constitutes an element of the subset $A$, it is considered that it is a break-down of the system. The selection of the subset greatly depends on the character of the given technical system, and, first of all, on the function fulfilled by the system and on the conditions in which it is to operate. The problem of reliability is often approached in a different way, especially when the phase space $X$ is just not well known. By way of example: the Boeing airplanes 720 , are undergoing the general overhauling after 2.000 hours of flight, independently of their condition.

On the basis of a great number of experiments concerning relia- 
bility of products of different kinds, we can see that the assumption about the function $x(t)$, that it is definitely determinating in its character, finds no confirmation. The analysis of wearing out of materials resulting from mechanical friction and blows, long use or from magnetic, electric, thermal, chemical and biological action, leads to more or less the same result. However, the goods produced in the same conditions, of the same raw-material and finding themselves in the same conditions, behave differently. We observe significant differences concerning the length of their life and change of their state, as well. The picture obtained explicitly resembles the course of a random process. Such a situation presently exists in all modern research concerning the theory of reliability.

Attention should be drawn to the fact that the character of processes $x(t)$, with which we are confronted in the analyses of concrete technical products, is but poorly known to us, particularly -regardless of the abundance of observations-concerning the significant number of problems connected with the distribution of length of their life, which are not yet examined universally.

We define the function of the distribution of the length of life of a certain technical products by $F(t)$. We shall denote the conditional density of the distribution of its life length by $\lambda(x)$, assuming that the product was in use during the period of time $x$.

In other words we assume:

$$
\lambda(x)=\frac{F^{\prime}(x)}{\mathrm{I}-F(x)}
$$

This function is known under the term "intensity of breakdown" which was borrowed from demography where it is called "intensity of mortality". A great number of analyses of both mechanical and radioelectrical products reveal that in all cases the diagram of the intensity of "breakdown" is of the following character: at the beginning the curve shows a sudden fall, then, during a long period, it preserves its constant value, and finally, in the third phase it increases constantly.

In radio-electronics, the first period is called "the period of annealing", in mechanical systems it is called the period of adaptation (breaking-in). The second period is that of normal work and the third one-of growing old. 
It is known that people's mortality represents precisely the same picture. Three characteristic periods are also observed in this respect: that of increased mortality among children, of normal mortality in the vigor of life and of increasing mortality in old age.

The above-mentioned analogy is fairly interesting, at least, from the point of view of experts in the field of the theory of reliability who should be acquainted with the results achieved by experts in demography. It would be also advantageous that specialists in demography be acquainted with the results achieved by experts in the field of the theory of reliability, their problems and experiences in the field of the theory of random processes arising in connection with the specific character of the theory of reliability.

I shall acquaint you now with a certain general result of the theory of reliability. Let us assume that an installation is composed of high number of elements, from among which each one undergoes a break-down independently of the others, and that the breakdown of each element leads to the break-down of the whole installation. Let, at the moment of break-down, the faulty element be immediately replaced by a new element of the same type.

We shall number the separate elements and we shall define by $X_{k}(t)$ the function which is equal to o since the moment o (this means since the moment the work has started) up to the first break-down $k$ of this element and, at the moment of its break-down, its increase up to unity.

Let us analyse the process

$$
\xi(t)=\sum_{k=1}^{n} x_{k}(t)
$$

where $n$ is the number of elements of the system.

It is obvious that the minimum value of the argument at which the function $\xi(t)$ increases, constitutes the break-down moment of the installation. The question arises whether, on the basis of general assumptions concerning the character of components, conclusions should be drawn on the general character of the course of the process taken as a whole or, at least on the time distribution since the moment at which the installation has started to work up to its first break-down. It appears that if the components are small, in comparison with the value of the sum, and independent, 
in the case of large $n$, the following approximate equation holds

$$
P[\xi(t+u)-\xi(u)=k] \approx \frac{[\Lambda(t+u)-\Lambda(u)]^{k}}{k !} e^{-[\Lambda(t+u)-\Lambda(u)]}
$$

for all $k=0, \mathrm{I}, 2,3, \ldots$ where $\Lambda(t)$ is a certain non-decreasing function.

The converse theorem also applies. For any non-decreasing function $\Lambda(t)$ such functions can be selected $x_{k}(t)$, for which the above-mentioned asymptotic equation holds. Let us observe that the probability of the fact that in the period from $u$ to $u+t$, the break-down will not take place -according to this statementequals

$$
\exp -[\Lambda(t+u)-\Lambda(u)]
$$

This general pattern can express and explain that the distributions of the length of life of technical installations, observed in numerous cases, are of exponential type, Weibull and many other types.

In connection with the fact that all technical installations lose, in the course of time, their capacity of work and that the process of wearing is observed with regard both to men and machines, the necessity is to formulate the theory of such random processes which would take into consideration the factor of wearing out of elements.

In recent years attempts have been made to create the theory of the wearing out elcments with the use of the results of research concerning the processes marked with an increasing intensity of break-downs.

The research in the field of wearing out elements permits to obtain effective results of a general character and in particular a good estimation of the average length of "life" of goods produced, of the probability of faultless operation and the like.

As far as the theory of reliability is concerned, research concerning the problems connected with the selection of an optimum organisation of work is fairly advanced, as well as that concerning the assessment of the moments of prophylactic control and repairs. The efforts of explorers are directed towards the achievement of the longest possible period of an installation's work, or of the best 
economic results, or, finally, of the lowest possible cost of the operation of installations. Attention should be given to the fact of the existence of similar problems with regard to people, too.

Thus we are faced with questions such as, for instance: How work should be organised in order that man could work with as little strain as possible? How the successive periods of work and leisure should be organised in order that the human organism wears out as little as possible with preservation of his full productivity?

At present, because of a great intensity of work in some specializations, the mentioned problems are important not only from an economic or medical point of view, but moreover, have a deep social meaning.

It is possible that the results obtained in the field of theory of reliability and concerning the optimum organisation of the prophylaxis of technical installations as well as the optimum length of their faultless operation, can find application to the problems of psychology of work and insurance, and thus may contribute to the prolongation of man's life and his work capacity.

A new branch of the theory of random processes can find its direct application to the solution of these problems, as it concerns the endeavours of finding the method of the optimum control for these processes. The complexity of such control consists in the fact that its possibilities are fairly limited and that we dispose of very poor data on the basis of which suitable decisions could be undertaken.

We are faced with such a type of problems in literally all fields of activity. An example to this end may be the control of a technological process which is influenced by random events. The question should be put in this respect, namely when the influence of this random events becomes so great that any further continuation of the process, without a reasonable directing it, becomes harmful or even dangerous. How should this phenomenon be prevented? Control of technological processes consists, first of all, in maintaining the production quality on a suitable level, in preventing its decrease below a defined level and simultaneously, in preventing a too - often stopping of production in order to regulate installations, replacement of equipment parts and changing of work conditions, etc. 
A random process which describes the course of production is usually analysed as a sum of two components, out of which one is stationary and the other is variable. Here we have the problem of establishing the optimum moment for the stopping of production, which presently is resolved in the case of very limited assumptions. The problem of prognosis concerning break-downs and control over the process consisting in the diminishing realiability of technical installations is, properly speaking, different from the abovementioned one only from the point of view of terminology. In the case of an increasing possibility of a break-down we intervene in the work of equipment by means of suitable prophylactic operations or by changing the condition of the given installation operation and removal of its dangerous state.

We shall see once again that problems concerning the control over random processes are of great importance for the control over the condition of human organisms, as well.

The theory of random processes finds itself in a flourishing period. A number of its problems still waits for solution. The theory is inseparably connected with different needs of practice and on its part it has an influence on the directions of practical analyses, inciting the thinking process of explorers and revealing new ideas and analogies. Almost seventy years ago, David Hilbert, one of the most outstanding mathematicians of the last century, expressed the following sentence: "Any scientific field can be developed only in the situation whenever new problems constitute the subject of research. Lack of new problems is synonymous with either vanishing or interruption of the development of the given science. Any action is also connected with a certain aim, thus mathematical research is connected with putting forward new problems."

The theory of random processes belongs to these fields of science which are not afraid of stagnation caused by unresolved problems or lack of connection with actual practical tasks. On the contrary, the existence and abundance of such connections assure the emerging of new and interesting problems, which, in turn, assure the permanent development of the theory of random processes and the long life of this theory. 